\title{
Binding Lifetime Based Signaling Cost Analysis of Multilayer MIPv6
}

\author{
Kushal Pokhrel ${ }^{*}$, Nitul Dutta ${ }^{2}$, Mrinal K. Ghose ${ }^{1}$, Hemali Vithalani² ${ }^{2}$ Hiren Kumar Deva Sarma ${ }^{3}$, \\ Zdzislaw Polkowski ${ }^{4}$ \\ ${ }^{1}$ Computer Science and Engineering Department, SMIT Majhitar, Rangpo, East Sikkim, India. \\ ${ }^{2}$ Computer Science and Engineering Department, MEFGI, Rajkot, India. \\ ${ }^{3}$ Information Technology Department, SMIT Majhitar, Rangpo, East Sikkim, India. \\ ${ }^{4}$ Jan Wyzykowski University, Skalnikow, St. No.6, B-59-101, Polkowice, Poland. \\ * Corresponding author. Tel.: +919733033173; email: kushal.p@smit.smu.edu.in \\ Manuscript submitted March 6, 2017; accepted June 26, 2017. \\ doi: $10.17706 /$ jcp.13.3.337-350
}

\begin{abstract}
An unbridled exchange of binding update messages have been observed in Mobile IP even for an infrequent movement of a mobile node. This not only makes the mobile node energy inefficient but also increases the signaling overhead. Even though binding lifetime and binding refresh cost are important parameters, they haven't received much attention from research community. This paper proposes algorithms to calculate the binding lifetime and binding refresh cost based on parameters like speed of mobile node, coverage area of mobility agents and session size in a TLMIPv6 environment. Analytical investigation shows that proposed algorithm not only reduces the signaling load of the network but also diminish the processing overhead at mobility agents.
\end{abstract}

Key words: Binding lifetime, binding refresh cost, mobility management, TLMIPv6.

\section{Introduction}

Internet on the move has become an integral part of our daily lives. As a consequence, Mobility Management (MM) of IP based networks is an essential part of networking research. To realize a realistic mobility model is not only critical but at the same time, a difficult aspect of systems that are designed to work in mobile environments such as MIPv6. That is why mobility management is a potential area of research gaining popularity among the researchers. Although, MIPv6 is comparatively well established, that addresses the mobility issue due to lack of address space and other such flaws, it is not suitable for next generation computing [1]. It leads to the design of IPv6 as next generation network protocol and subsequently MIPv6 is proposed for mobility management. Since its inception, many enhancements have been done to MIPv6 for performance improvement and to make the protocol more efficient for seamless mobility.

The prime driving metrics for analysis of MM protocols are handoff latency, signaling cost and tunneling cost. Researchers have emphasized on minimizing these parameters to make their suggestions acceptable globally. However, achieving minimized value of all these three parameters seems to be difficult and hence the trend is to select the optimized value for these parameters. In this paper we aim at analyzing the signaling cost of MM in IPv6 based network.

From the discussions above, it is certain that the performance of an IP protocol in mobile environments 
depend upon higher layer MM strategies. In an IP based protocol like MIPv6, a Binding Lifetime (BLT) is allotted to a mobile node when it transmits Binding Update (BU) information to its Home Agent (HA) and the concerned Correspondent Nodes (CN). If the BLT is too short then the binding update rate increases and if the BLT is too long that will occupy unnecessary space and valuable data resources. Therefore, it implies that the BLT has a definite impact on the signaling cost.

The rest of the paper is organized as follows. In Section 2, the related work will be summarized. In Section 3 , we will explain the analytical model which is the base for the calculation of the optimized binding lifetime. Section 4 will provide the detailed architecture and the underlying algorithms used for the experimentation. Section 5 will furnish the graphical results obtained, their analysis and comparison with the parameters of the existing protocols. Finally in Section 6, we will summarize our scheme of dynamical calculation of binding lifetime and discuss the scope of future research in the domain.

\section{Related Work}

A recent survey [1] shows that there is a strong need for a universal solution concerning the IP based mobility management protocols to be able to meet up with the demands of seamless high mobility of users and applications. This work also shows that there is a lot of scope in the research of hierarchical mobility management protocols such as HMIPv6 and their extensions for future IP networks.

Ample of research works have been reported on signaling cost analysis of IP based mobility management protocols. However, there are very few number of works [2]-[4] found that includes BLT and subsequent refresh cost for the analysis of signaling overhead. In this section, some of such related works that has resemblance to our work is discussed.

The work presented in [2] highlights the schemes for determining the BLT in order to make the mobile nodes energy efficient in a HMIPv6 environment. It has been stated that, if a mobile node remains in a particular service area for a long time then this information can be made to decrease the time interval for sending the location update messages. That is, if a mobile node additionally maintains a location log or profile, the information in the log may be used to estimate its BU Lifetime for that particular service area. However, the time of arrival and subnet visited by a node may not give the precise residence time under a subnet. Moreover, the communicated profile of mobile node to the Mobility Anchor Point (MAP) may be large due to the size of previous profile history. This in turn increases the overhead of Binding Management.

A separate study [3] shows that the signaling overhead in a HMIPv6 environment can be reduced by evaluating the binding cache to dynamically calculate the BLT of the Mobile Node (MN) between a maximum and a minimum value. A significant reduction in signaling cost is achieved by dynamically varying BLT. The default value of the BLT of the MN is set small and two different coefficients of BLT called as regional weight and local weight are made to vary as per the profile of the previous visits. This work considers the MN's residence time as an exponential distribution. However this work does not consider further division in hierarchy of mobility agents to handle different scale of mobility.

Similarly there are adequate literatures that examine the signaling cost of MM protocols. Most of them consider the BU messages, Binding Acknowledgement (BACK) messages and the cost of propagation of signals of such messages as the signaling overhead. But almost all of these protocols ignore the parameter called the Binding Refresh cost (BRC), which is directly associated the binding of MN with their Anchor Agents (AA). The basic criteria states that the validity of the temporary address assigned to the MN expires as the assigned BLT expires. If a MN stays within the same area after the expiry BLT then its location information needs to be refreshed with all the associated AAs. Needless to say, such refresh incurs similar cost as that of Binding Registration. It implies that BRC has equal impact as that of Binding Registration on signaling overhead in a scenario where most of the mobile nodes stay in the same area after the expiry of 
BLT. Therefore, selection of an optimized value of BLT is an important aspect for MM protocols. Despite of being a critical parameter, BRC is being overlooked in most of the research. There are limited numbers of literatures found [4]-[6] that consider BRC as one of the important criteria for signaling cost analysis. However there are many literatures that do consider BRC as an extraneous variable [7]-[9].

Looking at the importance of the BRC in signaling overhead analysis, in this paper, a thorough analysis of signaling cost considering BRC as its integral part is being carried out. Our work is influenced by [3], [9] and [10] however different in many aspects including the underlying MM architecture, method of optimized BLT computation and methodology adopted for analysis.

From the discussion carried out so far, it is seen that Mobile IP has been considered as the solution of a global mobility imparting MM across different radio devices and their subsidiaries. A fair amount of literatures have been published over the past few years that explore the feasible modification in the MIPv6 protocol for better system performances. Although it has been established that the Binding Update Lifetime has a telling impact on the performance of the system, but the estimation schemes of Binding Update Lifetime still remains a fairly unexplored research area.

\section{Analytical Model}

In this section we propose to determine BLT using parameters like Binding Update Rate (BUR), Session to Mobility Ratio (SMR) and Session Size $(\mu S)$. BUR is the ratio of velocity of MN to the coverage area of anchor agent (AA), SMR is the ratio of session arrival rate $\left(P_{n}\right)$ to the subnet changing rate $\left(R_{c}\right)$ and $\mu S$ is the average number of packets $\mathrm{MN}$ will receive in a particular session.

BUR has a significant impact on the BLT value. The BLT value increases if the speed of MN is increased. A higher speed of MN results in frequent binding update exchanges and that would in turn need lesser number of Binding Refresh (BR) messages. Therefore higher the value of binding lifetime, lower the number of BR messages. For a larger coverage area of AA, a fewer number of BU messages are required. Therefore for a MN to register to an AA having a larger coverage area, a smaller BLT value is required in the BU message exchanged between the MN and AA. Such MNs requiring continual services with the current Care-of-Address ( $\mathrm{CoA})$ need to send higher number of BR messages. Thus, smaller the BLT, higher the number of BR messages exchanged. BUR can be determined with help of (1).

$$
\mathrm{B} \mathrm{UR}=r / v
$$

where $v$ is the velocity of the MN and $r$ is the radius used to determine the coverage area of AA. For simplicity of mathematical modeling, the radius of the coverage area has been used instead of diameter as they are both linearly related and hence do not significantly affect the results.

SMR also impacts the BLT value in the BU messages exchanged between MN and AA. In general, a random, mutually independent message arrival can be modeled as a Poisson process with an average session arrival rate $P_{n}$. The rate of change of subnet for a MN $\left(R_{c}\right)$ can be realized as the coefficient of mobility of MN which uses the Fluid Flow Mobility Model [11]. SMR can be calculated using (2). It is important to consider SMR to calculate the BLT for providing uninterrupted services to MN. The reason being frequent updates in the routing table of AA in case of increasing session arrival rates when MNs are frequently changing their subnets.

$$
\mathrm{SMR}=\frac{P_{n}}{R_{c}}
$$

where

$$
p_{n}=\frac{e^{-\lambda T^{*}}(\lambda T)^{n}}{n !}
$$


and

$$
R_{c=} \frac{\pi v}{4 r}
$$

Here $T$ is the time interval considered for session arrival rate, $\lambda$ is average arrival rate, $n$ is number of packets arrived without any assumption. $\mu S$ is directly proportional to the BLT in a BU message exchanged between MN and AA. A fair conclusion that can be drawn from the above discussion is that the BUR has better impact in optimizing BLT than SMR. The equation for determining the optimized binding lifetime is given as follows:

$$
T_{\text {life }}=W_{1} * \mathrm{BUR}+W_{2} * \mathrm{SMR}+W_{3} * \mu S
$$

where $W_{1}, W_{2}$ and $W_{3}$ are the weight variables assigned to the BUR, SMR and $\mu S$ respectively.

\section{Architecture}

\subsection{System Model}

The aspect of determining optimized binding lifetime is defined in this paper by using Three Layer MIPv6 (TLMIPv6) [12] network as shown in Fig. 1. Basically three layer architecture split the network into to two parts: backbone and inside domain. The inside domain is further divided into three parts: global, regional and local domain.

In TLMIPv6 architecture whenever the MN changes its location form old AR to new AR, the MN needs to send a BU message to MAP under which the new AR is located. In the similar manner, when MN changes its location form old MAP to new MAP, the MN needs to send a BU message to Regional Mobility Anchor Point (RMAP) which manages the new MAP. The same is applicable for a MN moving from one RMAP to other RMAP i.e., it needs to send BU to Global Mobility Anchor Point (GMAP) which manages the new RMAP. Thus $\mathrm{BU}$ to home agent and corresponding node is required only when MN moves form old GMAP to new GMAP which will drastically reduce the signaling load of the network.

BLT is amount of time after which MN needs to send BR message to its mobility agent and CN. More number of BR messages needs to be exchanged when the value of BLT is small and vice versa. BLT is specified whenever MN sends BU message to $\mathrm{CN}$ and mobility agents such as HAs and AAs. Whenever MN changes its location it needs to send BU message to its mobility agents and CN. If the MN stays within the same area for more than the specified BLT then it needs to send BR to mobility agents and CNs. The purpose of sending BR message is to re-register with the current location and current CoA.

\subsection{Operational Model}

The CoAs are used for identifying current location of MN [13]. The CoA first acquisition process as depicted in Fig. 2 includes following steps:

Step 1: AR (Access Router) broadcasts its routing advertisement messages in its network. In a foreign network a $\mathrm{MN}$ acquires its $\mathrm{CoA}$ form routing advertisement message.

Step 2: After acquiring CoA, MN sends Duplicate Address Detection (DAD) request to AR to examine the uniqueness of CoA.

Step 3: AR responds DAD request to $\mathrm{MN}$ as successful if CoA is unique, otherwise unsuccessful if CoA is duplicate. The above steps are until a successful CoA has been acquired by the visiting MN.

In IPv6, MNs obtain their CoA by using address auto configuration method which reduces the handoff latency of the network [13]. The handoff process depicted in Fig. 2 shows the global updating process. After 
the global updating, the network requires some regional and local updating also. Handoff and CN update process includes following steps:

Step 1: After the successfully acquiring a unique CoA, the MN stores it and starts binding update process. The MN sends BU message to HA which contains its current CoA.

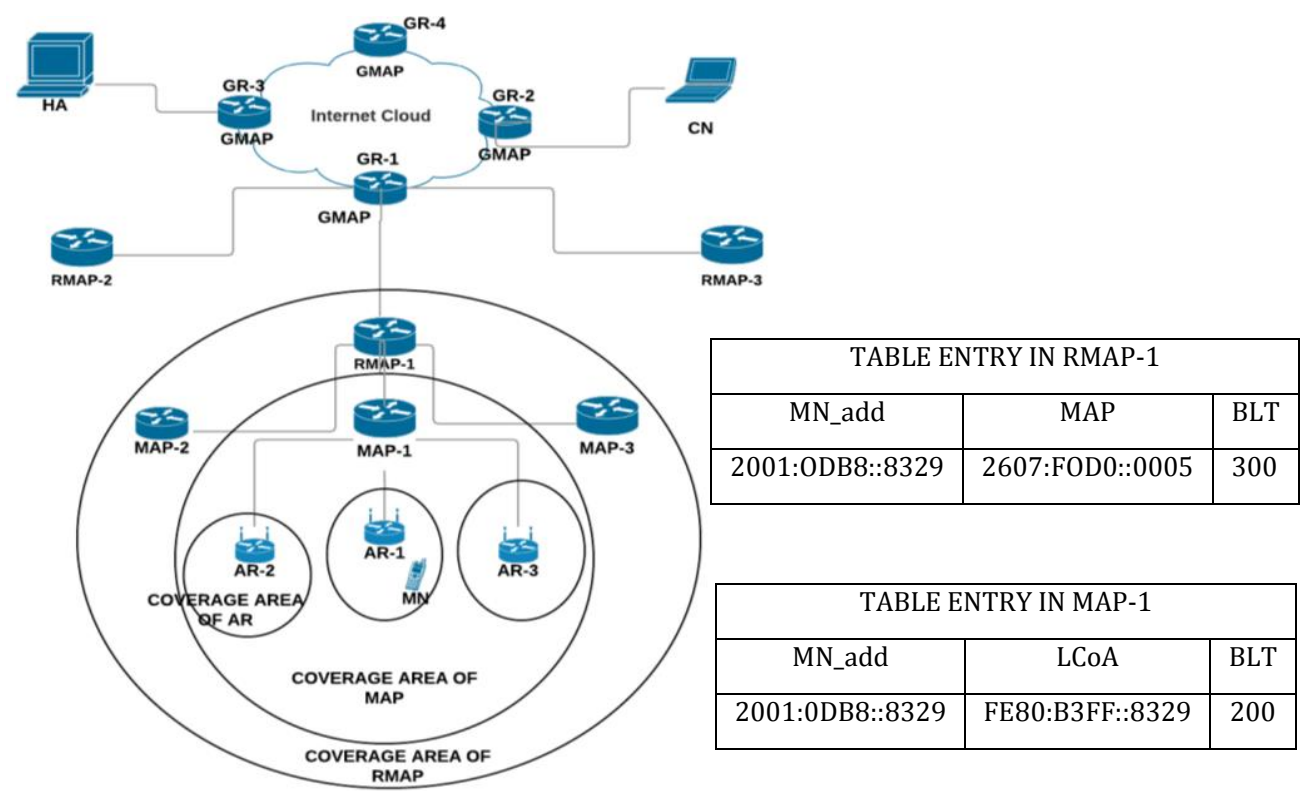

Fig. 1. Three layer MIPv6 network model.

Step 2: HA stores that CoA in its database and bind CoA with MN's permanent address. HA acknowledges the BU message by sending a BACK message.

Step 3: If MN is associated with any $\mathrm{CN}$ then later on MN can notify $\mathrm{CN}$ about its new location.

Step 4: $\mathrm{CN}$ acknowledges that message by sending a BACK message.

The binding refresh process includes following steps:

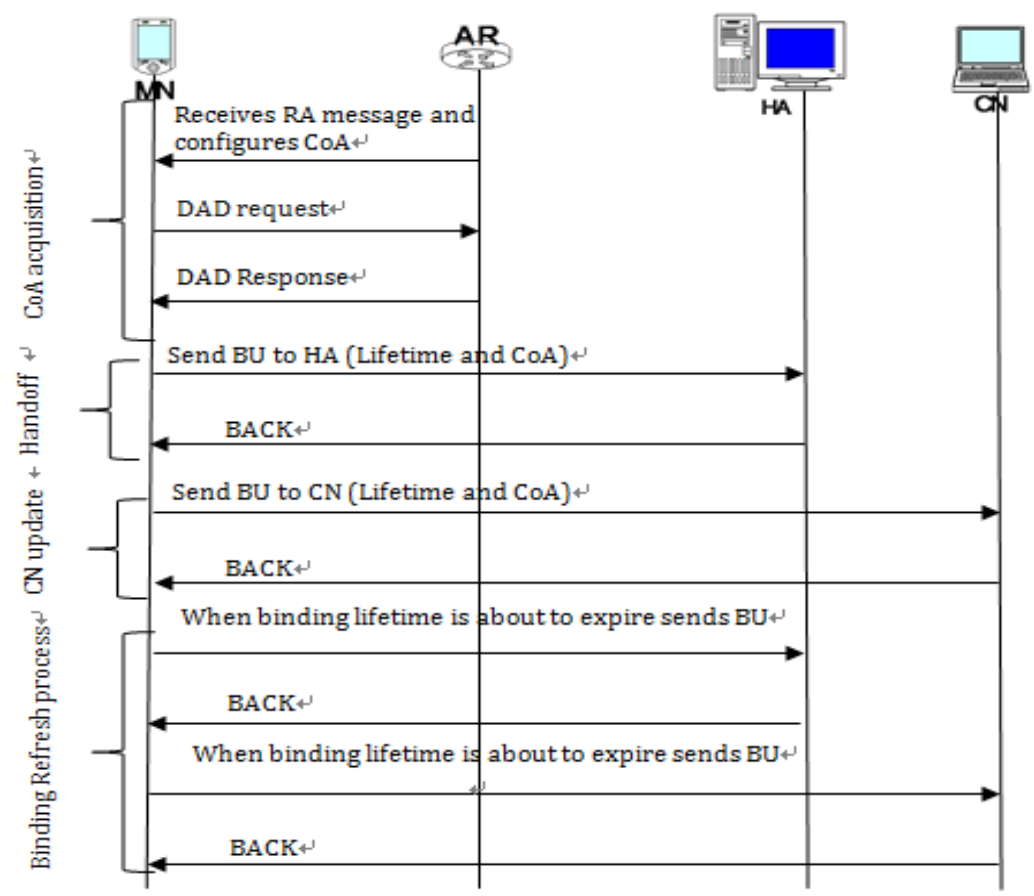

Fig. 2. Mobility in MIPv6 network. 
Step 1: Whenever binding lifetime is about to expire MN sends BU message to its HA to refresh the binding information which allows HA to trace its MN at any instant.

Step 2: HA restores the binding information in its database and acknowledges the BU message by sending a BACK message.

Step 3: If $\mathrm{MN}$ is associated with $\mathrm{CN}$ then $\mathrm{MN}$ can send $\mathrm{BU}$ message to $\mathrm{CN}$ to refresh binding information.

Step 4: $\mathrm{CN}$ responds to that BU message by sending a BACK message.

\subsection{Binding Refresh Scenario}

As a demonstration shown in Fig. 3, each AA and MN maintains routing table which includes information such as address of $\mathrm{MN}$, address of $\mathrm{AA}$ where $\mathrm{MN}$ is located and BLT value. AA's are required to send BR message when BLT is about to expire. When BLT value specified in MN's routing table is near to expire, the MN needs to send BR message to the MAP. The purpose of sending BR message is to notify the MAP that MN is still in its coverage area. MAP provides acknowledgment of BR message by sending BACK message. When the BLT at MAP is about to expire, the MAP needs to send a BR message to RMAP to provide information that $\mathrm{MN}$ is still in its coverage area. RMAP acknowledges that message by sending a BACK message. In same manner whenever BLT at RMAP is about to expire, RMAP needs to send BR message to GMAP to notify that MN is still in its coverage area. GMAP acknowledges that BR message by sending a BACK message.

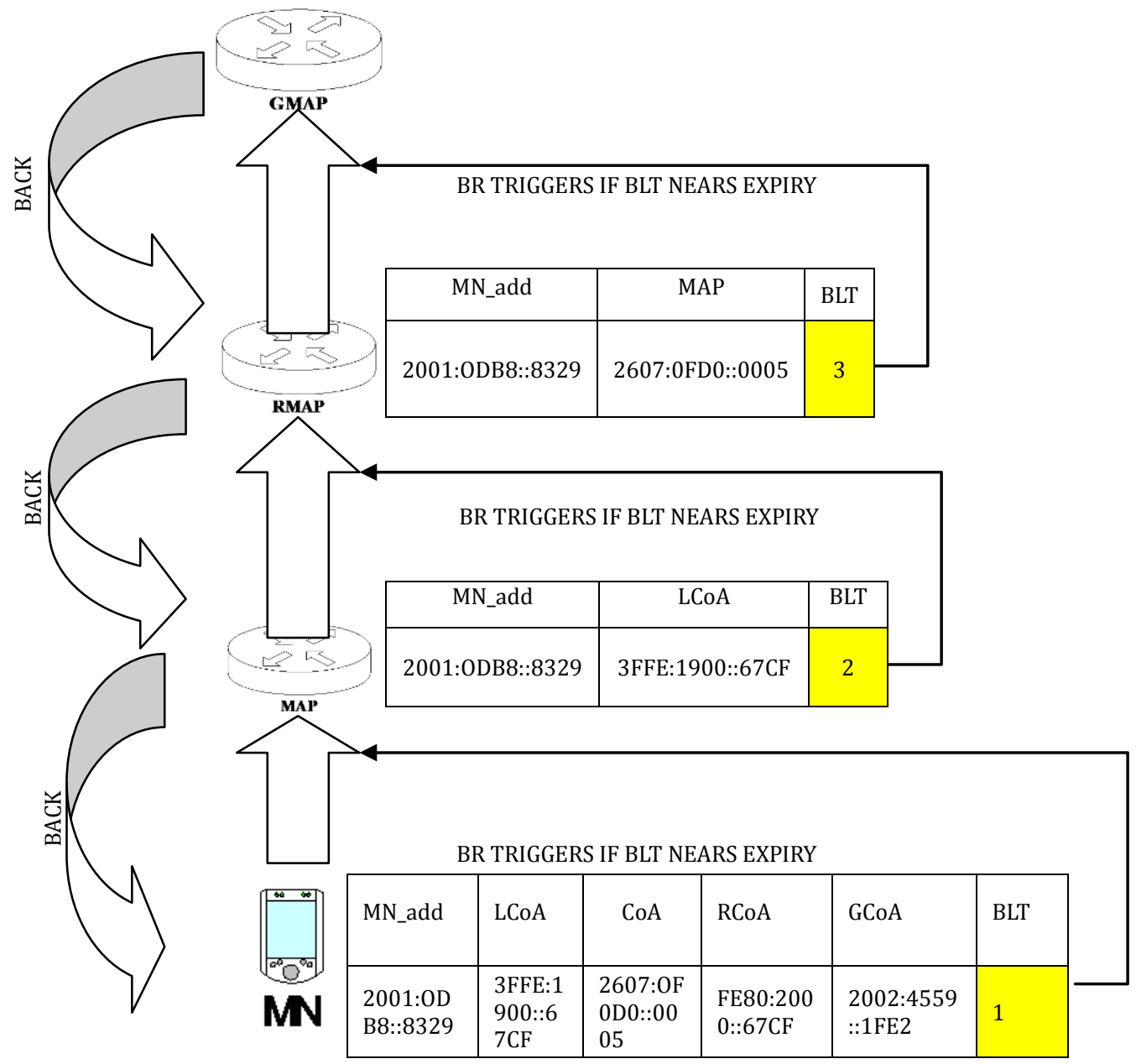

Fig. 3. Binding refresh scenario in MIPv6.

\section{The Algorithm}


This section proposes the algorithms for BU messages and BR messages for determining the optimized binding lifetime $\left(T_{\text {life }}\right)$. Table 1 lists the parameters used in our algorithm. As discussed in the previous sections, whenever a MN changes its location, it requires sending BU message to its mobility agents and CN. BU message essentially consists of location information and BLT. Fig. 4 shows an algorithm for binding update message that comprises of two processes in each case. The first one is to calculate binding lifetime $\left(T_{\text {life }}\right)$ and the other is to send BU to appropriate mobility agents and $\mathrm{CN}$.

The proposed algorithm for calculating the optimized BLT is shown in Fig. 5. As discussed in the earlier sections, the optimized value of BLT is depends on SMR, BUR and $\mu S$. This algorithm contains three procedures. The first one is "Calculate BUR" used to determine BUR that uses two parameters for its calculation which are $v$ and $r$. The second procedure is "Calculate $\operatorname{SMR}(\lambda, T, n, v, r)$ " that determines SMR as the ratio of session arrival rate and subnet changing rate. This procedure first calculates the value of $\mathrm{Pn}$ and $R_{c}$ and then determines the ratio of the two. The third procedure "Calculate $T_{\text {life }}$ " is used for the calculation of optimized BLT as a function of BUR, SMR and $\mu S$. In the joint event when the BLT nears expiry, and the $\mathrm{MN}$ is still in the same location then the MN need to re-register with the current location by sending BR message. The algorithm for determining the BR message for this case has been demonstrated in Fig. 6 .

Table 1. List of Parameters Used

\begin{tabular}{|c|l|}
\hline Symbol & \multicolumn{1}{|c|}{ Parameters } \\
\hline$T_{\text {life }}$ & Optimized binding lifetime (s) \\
\hline$n$ & $\begin{array}{l}\text { Arrival rate of } \mathrm{N} \text { packet without any assumption } \\
\text { (packets/session) }\end{array}$ \\
\hline$T$ & Time interval (s) \\
\hline$\lambda$ & Average number of arrival rate (packets/session) \\
\hline$r$ & Radius of the coverage area of AA (m) \\
\hline$v$ & Speed of MN (m/s) \\
\hline
\end{tabular}

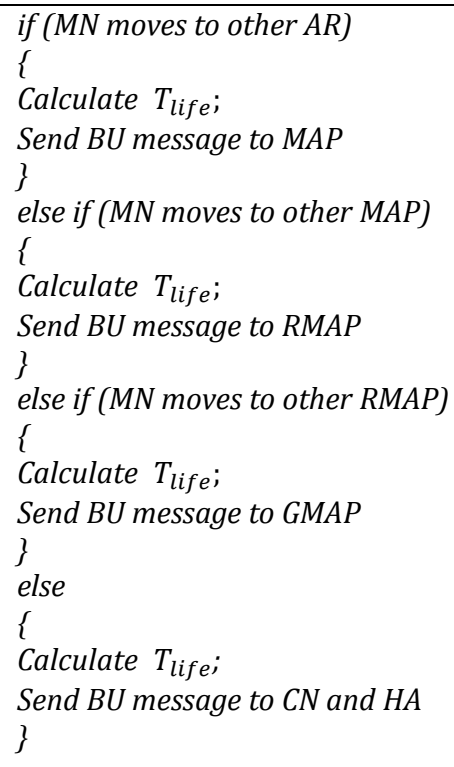

Fig. 4. Algorithm for BU message. 


$$
\begin{aligned}
& \text { Calculate } \operatorname{BUR}(v, r) \\
& \left\{\quad B U R=\frac{v}{r}\right. \\
& \} \\
& \text { Calculate } \operatorname{SMR}(\lambda, T, n, v, r) \\
& \{ \\
& P n=\frac{e^{-\lambda T} *(\lambda T)^{n}}{n !} \\
& R_{c=} \frac{\pi v}{4 r} \\
& \text { SMR }=\frac{P n}{R c} \\
& \} \\
& \text { Calculate } \quad T_{\text {life }}(B U R, S M R, \mu S) \\
& \left\{\quad T_{\text {life }}=W_{1} * B U R+W_{2} * S M R+W_{3} * \mu S\right.
\end{aligned}
$$

Fig. 5. Algorithm for determining the optimized binding lifetime.

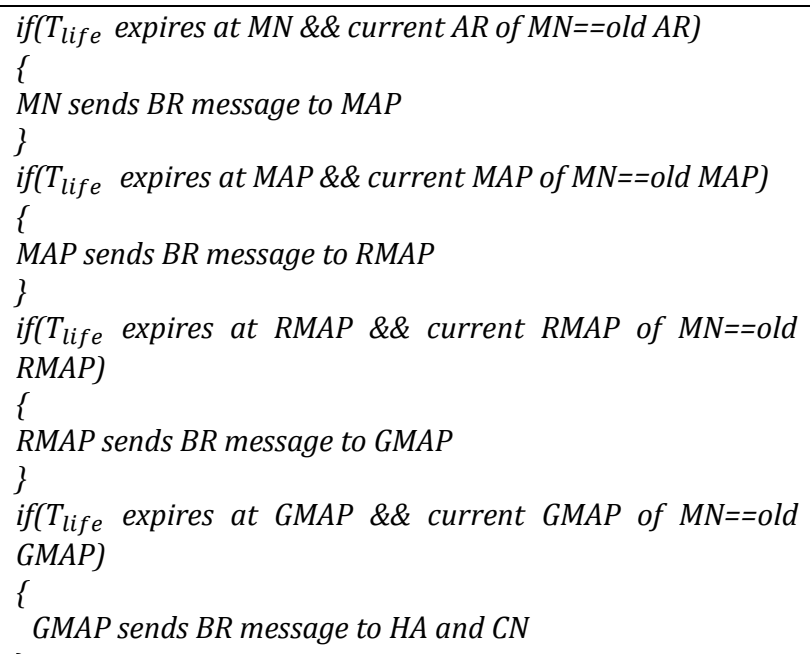

Fig. 6. Algorithm for binding refresh message.

This algorithm demonstrates the discrimination between local mobility, regional mobility and global mobility for the calculation of the optimized value of BLT. Hence every time the BLT is about to expire whilst MN being still in the coverage area of any of the mobility agents, then it needs to send BR message to its appropriate mobility agents.

\section{Results}

This section shows the obtained experimental results of BLT that using the algorithms presented in the previous section. Table 2 shows the values of parameters used during the experiment. We also present the impact of the parameters used for the determination of BLT and comparison of existing approach and the proposed approach.

As shown in (1), BUR is dependent on speed of MN and coverage area of AAs. Fig. 7 illustrates the impact of BUR on the BLT value and hence shows the impact of speed of MN and coverage area of AAs on the BLT value. For example, if $r=10 \mathrm{~m}$ and $v=23 \mathrm{~m} / \mathrm{sec}$ then $T_{\text {life }}=101.022 \mathrm{sec}$. This means that the MN needs to send a BR message after every 101 seconds. Here $\lambda, T, \mathrm{n}$ and $\mu S$ are taken as 1 packet/session, 10sec; 17packets/session and 300packets/session respectively. From Fig. 7 we can conclude that as $v$ increases, $T_{\text {life }}$ decreases. We can also reinstate that higher speeds of MN require exchanges of more number of BU 
messages which in turn would lead to exchange of less number of BR messages. Hence a larger $T_{\text {life }}$ value reduces the number of BR messages exchanged. Also we observe that the increase in $r$, results in the increased $T_{\text {life }}$ value, as larger coverage area requires exchange of lesser number of BU messages. This would in turn require sending more number of BR messages for the MN to be continuing the services with current CoA. Therefore it follows that a small binding lifetime value leads to a significant escalation in the number of BR messages exchanged.

Table 2. List of Parameters Used

\begin{tabular}{|c|c|}
\hline Parameter & Value / Range \\
\hline$v$ & $5-30(\mathrm{~m} / \mathrm{sec})$ \\
\hline$r$ & $3.17-10(\mathrm{~m})$ \\
\hline$\mu S$ & $50-1000($ packets $/ \mathrm{sec})$ \\
\hline$W_{1}$ & 0.44 \\
\hline$W_{2}$ & 0.22 \\
\hline$W_{3}$ & 0.33 \\
\hline
\end{tabular}

Fig. 8 shows the impact of SMR on $T_{\text {life }}$. Here the values considered for $r, v, T, n$ and $\mu S$ are $17 \mathrm{~m}, 25$ $\mathrm{m} / \mathrm{sec}, 20 \mathrm{sec}, 10$ packets/session and 350 packets/session respectively. The results obtained shows that a lower value of the packet arrival requires to set a small binding lifetime value and vice-versa in order to reduce the signalling load of network thereby eliminating the number of unnecessary exchanges of BR messages.

Fig. 9 demonstrates the impact of $\mu S$ on $T_{\text {life }}$. Here the values considered for $r, v, T, n$ and $\lambda$ are $9 \mathrm{~m}, 15$ $\mathrm{m} / \mathrm{sec}, 6 \mathrm{sec}, 17$ packets/session and 1packet/session respectively. It is evident from Fig. 9 that if the value of $\mu S$ is small then it is required to have a smaller value of $T_{\text {life }}$. This means that if the packet arrival rate is slow, it is required to set a smaller expiry time of the visiting MN's record in the routing table of the underlying mobility agent. Thus small binding lifetime allows MN to send BR message at smaller intervals. In a situation where $\mu S$ is large then a large $T_{\text {life }}$ value imposes less signaling load on the network by reducing excessive exchange of $\mathrm{BR}$ messages.

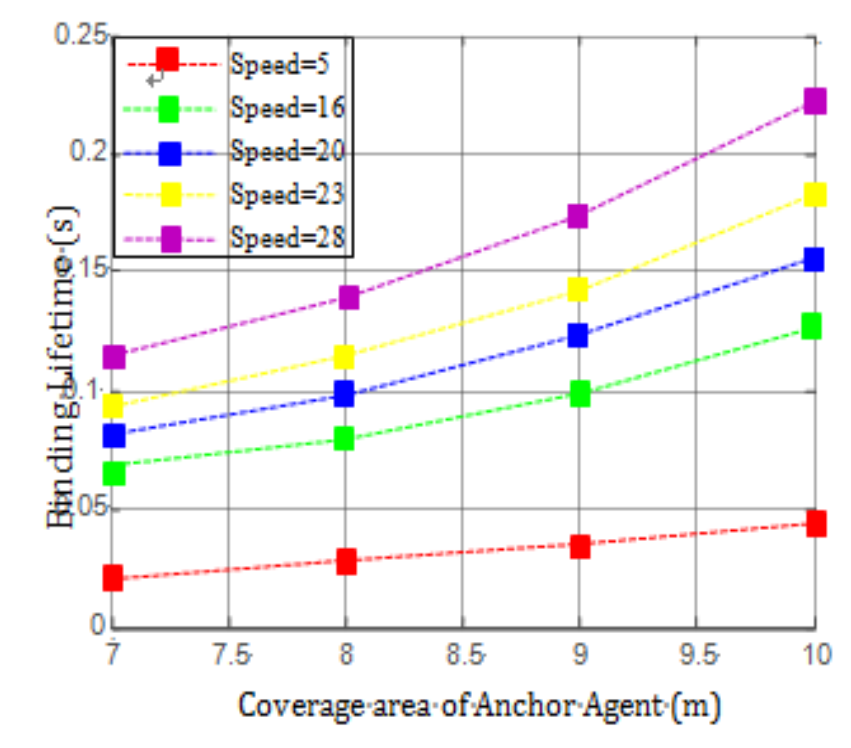

Fig. 7. Impact of coverage area and speed in binding lifetime. 


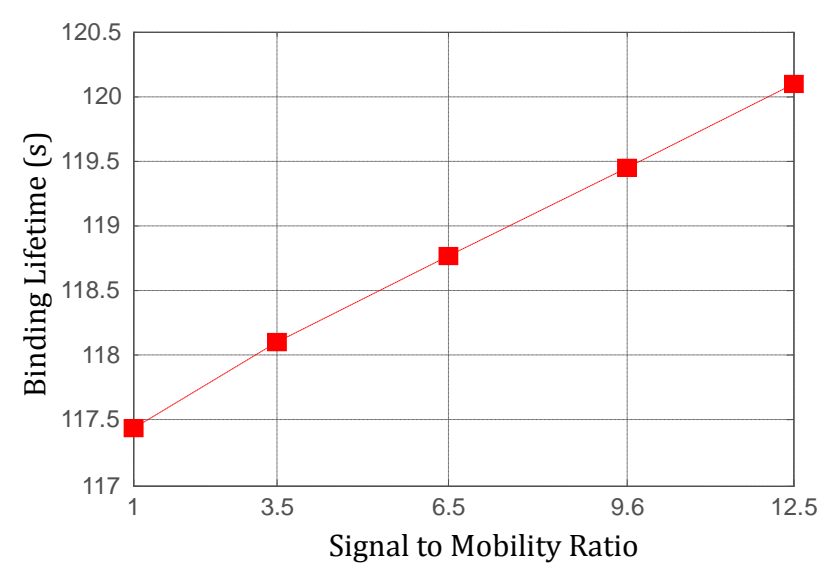

Fig. 8. Impact of SMR on binding lifetime.

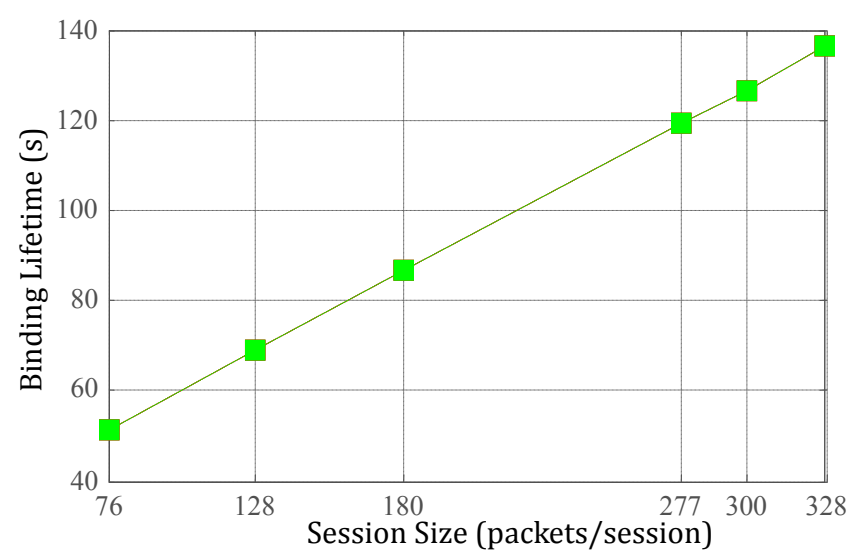

Fig. 9. Impact of session size on binding lifetime.

Fig. 10 illustrates the comparison between the lifetime values of proposed approach with that of the existing approach in MIPv6. As the binding lifetime specified in [13] is independent of any mobility parameters of MN, thus a MN in MIPv6 network would have a $T_{\text {life }}$ value that is independent of its speed, the coverage area of mobility agents and other essential parameter such as session arrival rate and session size. The comparison shows that the optimized binding lifetime $\left(T_{\text {life }}\right)$ value is conflicting for different mobility parameters such as speed, session arrival rate and session size. Thus we can conclude that the proposed approach dynamically determines the values of binding lifetime.

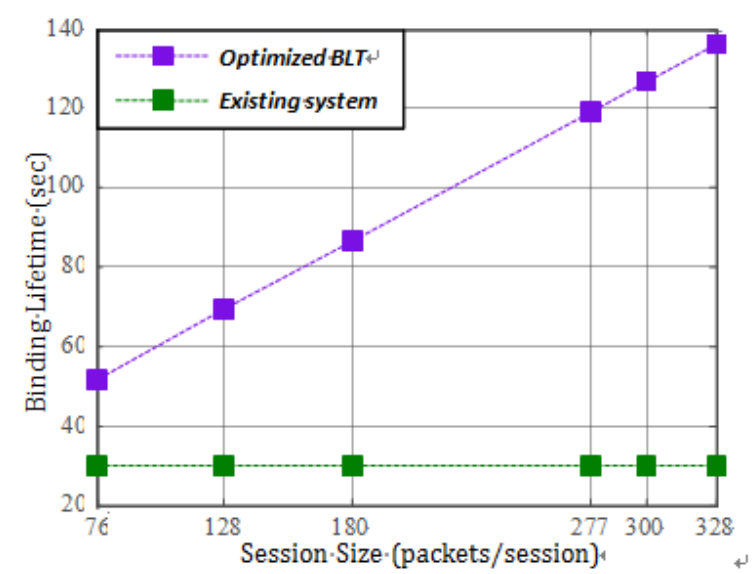

Fig. 10. Comparison of network with optimized binding lifetime and without optimized binding lifetime. 
In MIPv6 network binding lifetime for MN is between 1 second to 7 minutes [2]. The graphs in Fig. 12, 13 and 14 shows the comparison between signaling load of the network with optimized BLT to that without optimized BLT. The binding lifetimes for the experiment are taken as $3 \mathrm{sec}, 80 \mathrm{sec}$ and $200 \mathrm{sec}$ respectively. Fig. 11 shows that the network with optimized BLT causes less signaling load on the network as compared to that without optimized BLT. Also, the dynamic behavior of optimized BLT imposes less signaling load on the network by reducing the number of binding related messages. The graph presented in Fig. 13 shows that both the networks (network with optimized BLT and that without optimized BLT) impose same signaling load for a residential time of $300 \mathrm{sec}$. But if the MN stays in the same location for more than 300 second in the network without optimized binding lifetime, then MN causes more singling load. This is because of the exchange of a significant number of BR messages. Fig. 14 demonstrates the scenario where binding lifetime of MIPv6 network is assumed as 200 seconds. In this situation optimized binding lifetime approach imposes more signaling load for residential time of MN up to $180 \mathrm{sec}$ but beyond that it imposes significantly less signaling load on the network.

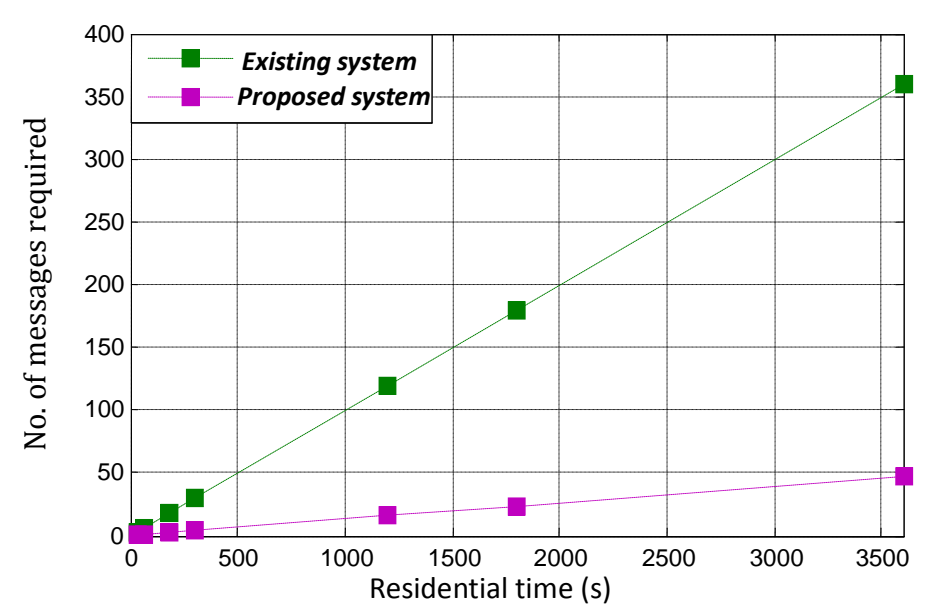

Fig. 11. Number of messages required vs. residential time.

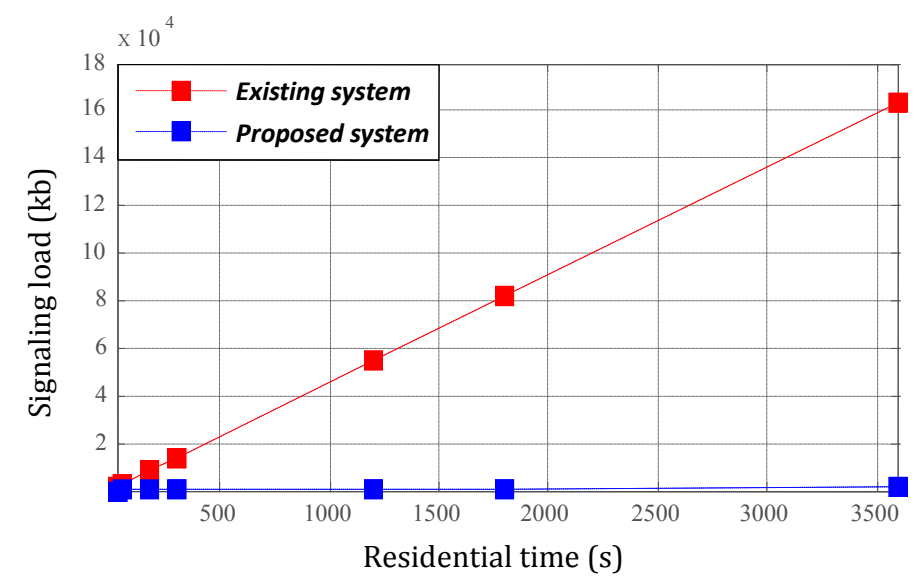

Fig. 12. Signaling load of the network with optimized binding lifetime vs. binding lifetime as 3 sec.

Thus we can say that our proposed optimization technique of binding lifetime of a visiting MN in a foreign network has the potential to become a major steppingstone towards handling the high data traffic volumes which is expected to boost in real time in near future towards the development of next generation networks [14]. The optimized value of binding lifetime aims to avoid the overwhelming signaling load that would result from extensive exchanges of binding related messages between the visiting mobile node and the 
associated mobility agents.

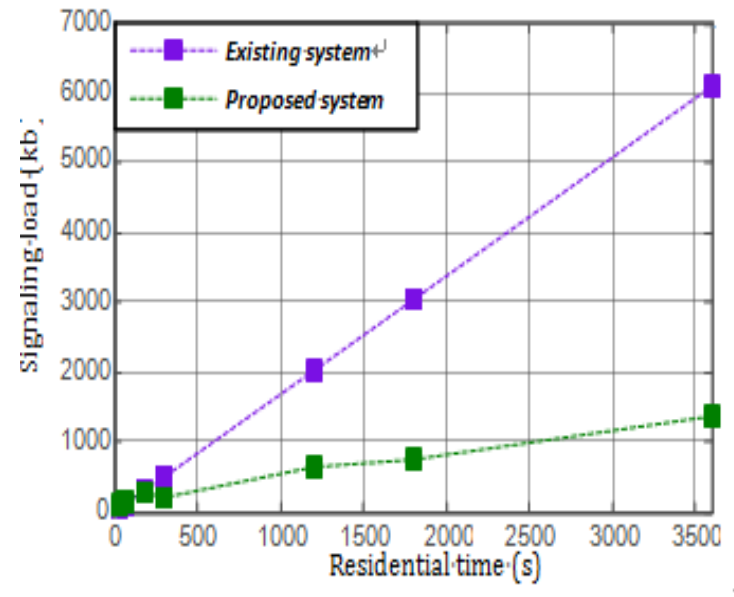

Fig. 13. Signaling load of the network with optimized binding lifetime vs. binding lifetime as 80 second.

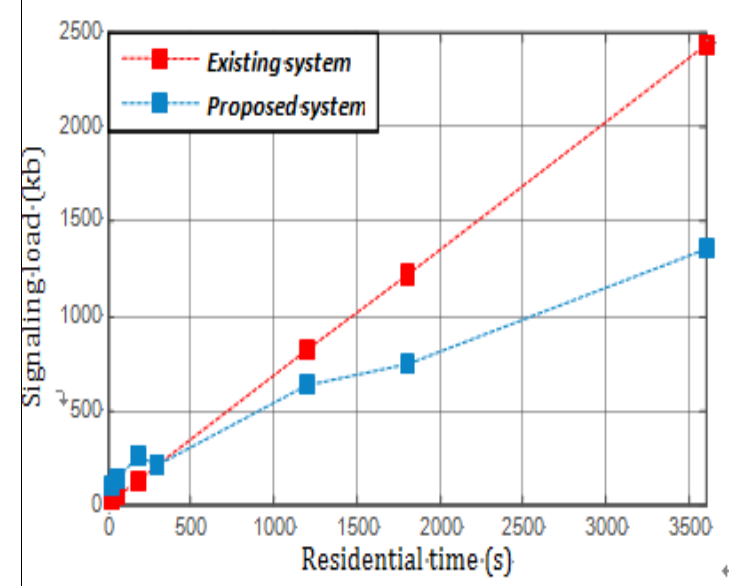

Fig. 14. Signaling load of the network with optimized binding lifetime vs. binding lifetime as $200 \mathrm{sec}$.

\section{Conclusion}

A frequent and unchecked emission of binding related messages in a Mobile IP network brings about unnecessary increase in the overall signaling cost. In this paper we propose the approach for dynamically determining optimized binding lifetime in context of layered IPv6. We have considered the TLMIPv6 protocol that divides the mobility agents in a hierarchical manner for our analysis. By doing so, we could discriminate between different scales of mobility of a visiting mobile node and the respective behavior of the corresponding mobility agents. In our work, we have assumed that calculation of binding lifetime and binding refresh cost are detrimental parameters for evaluation of the signaling cost of any mobility management protocol. We have proposed mathematical models and algorithms to calculate the binding lifetime and binding refresh cost based on speed of mobile node, coverage area of mobility agents and session size in a TLMIPv6 environment. The graphical results reveal that our model reduces the signaling load of the network and also shrink the dispensation of signaling overhead at mobility agents. In this work we have only focused on the mathematical modeling for the optimization of binding lifetime assuming a Fluid Flow Model for the arrival of mobile nodes in a foreign network. This work can be extended for optimization of binding lifetime based on other mobility models. In this work we have not considered the Handoff Latency in our analysis. Therefore a similar approach, an optimal reduction in Handoff latency may also modeled which can be a potential area in continuing research in the domain of Mobile IP. Also, software simulation of the mathematical model may also be considered as another extension of this work.

\section{References}

[1] Wozniak, J. (2016). Mobility management solutions for current IP and future networks. Telecommunication System, 61, 257-275.

[2] Yang, S. O., Kim, S., \& Hwang, C. S. (2006). Profile-based lifetime determination schemes for mobility management in HMIPv6. Journal of Information Science and Engineering, 22(3), 659-674.

[3] Myung-Kyu, Y., \& Chong-Sun, H. (2003). Dynamic allocation of binding update lifetime for cost reduction of location update in mobile IPv6. Proceedings of the International Conference on Wireless Networks. ICWN (pp. 182-188). Las Vegas, USA: Nevada. 
[4] Shohrab, M. H., Mohammed, A., \& William, I. (2010). Cost analysis of mobility entities of hierarchical mobile IPv6. Proceedings of the Military Communications Conference. MILCOM, IEEE (pp. 2280-2285). California, USA: San Jose.

[5] Shohrab, H., \& Mohammed, A. (2013). Cost analysis of mobility protocols. Telecommunication Systems, 52(4), 2271-2285.

[6] Wu, Z. D. (2003). An approach for optimizing binding lifetime with mobile IPv6. Proceedings of the 28th Annual IEEE International Conference in Local Computer Networks (pp. 82-88). Bonn, Germany: Königswinter.

[7] Nitul, D., \& Saha, M. (2014). Multilayer hierarchical model for mobility management in IPv6: A mathematical exploration. Wireless Personal Communications, 78(2), 1413-1439.

[8] Sanchez, I., Engin, Z., Antonio, O., Serdar, T., Utku, Y., \& Carlos, B. (2016). Mobility management: Deployment and adaptability aspects through mobile data traffic analysis. Computer Communications, 95, 3-14.

[9] Nitul, D., \& Saha, M. (2010). Cost analysis of a three layered MIPv6 (TLMIPv6) mobility model and HMIPv6. International Journal on Computer Science and Engineering, 2(1), 36-46.

[10] Hemali, V., \& Nitul, D. (2014). An analysis of optimized binding lifetime for mobility management in layered IPv6. Proceedings of IEEE International Conference on Computational Intelligence and Computing Research (pp. 1-5). India: Coimbatore.

[11] Nitul, D., \& Saha, M. (2012). Fluid flow and random walk mobility model for wireless mobile network research: A review. Advanced Materials Research, 4486-4494.

[12] Nitul, D., Saha, M., Abusafi, M., \& Kushal, P. (2012). Three layer MIPv6 (TLMIPv6): A new mobility management protocol for IPv6 based network. Proceedings of IEEE International Conference on Computational Intelligence and Computing Research (pp. 1-7). India: Coimbatore.

[13] Charles, P., David, J., \& Jari, A. (2011). Mobility Support in IPv6.

[14] Safa, Sadiq., Norsheila, B., Kayhan, Z., \& Jaime, L. (2014). Advanced mobility handover for mobile IPv6 based wireless networks. The Scientific World Journal. Retrieved from http://accurapid.com/journal/17prof.htm
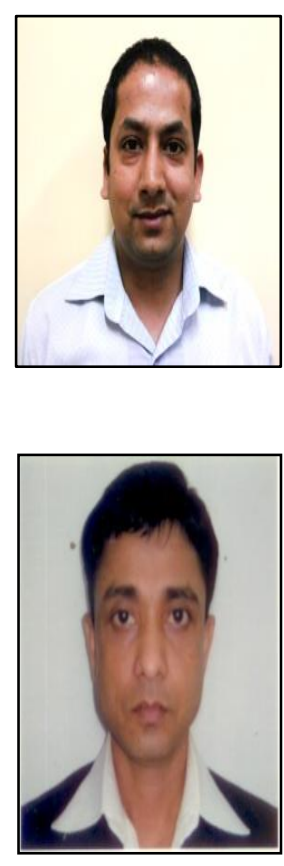

Kushal Pokhrel is currently associate professor in the Department of Electronics and Communication Engineering, Sikkim Manipal Institute of Technology, Sikkim, India. He received his B.E. degree in electronics and telecommunication engineering from Mumbai University in 2003 and M.Tech in digital electronics and advanced communication from Sikkim Manipal University in 2005. He is pursuing his Ph.D in engineering at Sikkim Manipal University. His current research interests are in the areas of IPv6 and mobility management protocols.

Nitul Dutta is currently professor in the Department of Computer Science and Engineering, MEFGI, Rajkot. He received his B.E. degree in computer science and engineering from Jorhat Engineering College, Assam in 1995 and M.Tech in information technology from Tezpur University Assam in 2002. He completed his Ph.D. in engineering in the field of mobile IPv6 at Jadavpur University in 2012. He has published more than twenty-five research papers in different international journals, referred international and national conferences of repute. His current research interests are in the areas of mobility management architecture and protocols in IPv6 based network and cognitive radio network. 


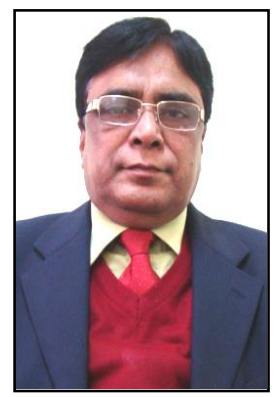

M. K. Ghose is currently professor in the Department of Computer Science \& Engineering at Sikkim Manipal Institute of Technology, Sikkim, India. Prior to this, he worked in the internationally reputed R \& D organization ISRO - during 1981 to 1994 at Vikram Sarabhai Space Centre, ISRO, Trivandrum in the areas of mission simulation and quality \& reliability analysis of ISRO launch vehicles and satellite systems and during 1995 to 2006 at Regional Remote Sensing Service Centre, ISRO, IIT Campus, Kharagpur in the areas of RS \& GIS techniques for the natural resources management. His areas of research interest are data mining, simulation \& modeling, network, sensor network, information security, optimization \& genetic algorithm, digital image processing, remote sensing \& GIS and software engineering. Ghose chaired a number of international/national conference sessions. He has conducted quite a number of seminars, workshop and training programmes in the above areas and published 126 technical papers in various national and international journals in addition to presentation/ publication in several international/ national conferences. Till date, he has produced $8 \mathrm{Ph}$.Ds and research assistance given for 2 Ph.Ds. Presently 8 scholars are pursuing Ph.D work under his guidance. Ghose is having 8 sponsored projects worth of 1 crore. Ghose also served as technical consultant to various reputed organizations like IIT, chennai, IIT kharagpur, WRI, tricy, scimst, keltron, HLL, trivandrum.

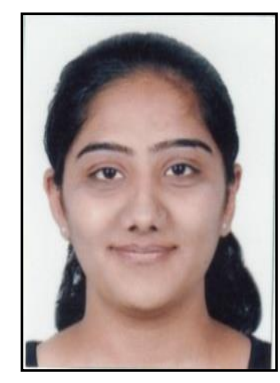

Hemali Vithalani is currently assistant professor in the Department of Computer Science and Engineering, MEFGI, Rajkot. She received her B.E. and M.Tech from Gujarat Technolological University. She is pursuing her PhD in engineering at MEFGI, Rajkot. Her current research interests are in the areas of IPv6 and mobility management protocols.

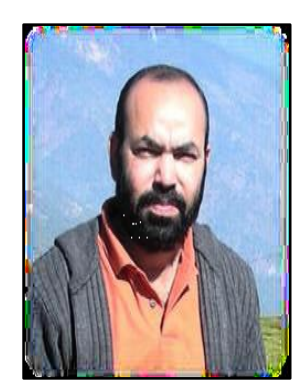

Hiren Kumar Deva Sarma is currently a professor in the Department of Information Technology, Sikkim Manipal Institute of Technology, Majhitar, East Sikkim, India. He has expertise in the field of computer networks, distributed systems and security of information. He has academic experience of over 20 years and has published many papers in international journals and conferences in the field of computer networks, distributed systems and security of information. He currently heads the information technology department in Sikkim Manipal Institute of Technology.

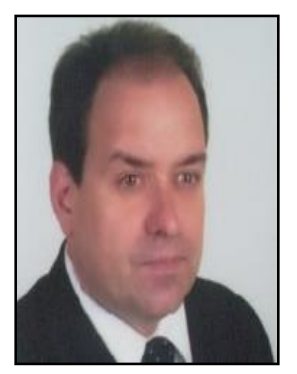

Zdzislaw Polkowski is currently a professor in Jan Wyzykowski University, Skalnikow, St. No.6, B-59-101, Polkowice, Poland. He is a distinguished professor in the field of computer networking, database management and economics. He has more than 100 publications in reputed journals and conferences to his credit. 